\title{
On Nonlinear Nonlocal Systems of Reaction Diffusion Equations
}

\author{
B. Ahmad, ${ }^{1}$ M. S. Alhothuali, ${ }^{1}$ H. H. Alsulami, ${ }^{1}$ M. Kirane, ${ }^{2}$ and S. Timoshin ${ }^{3}$ \\ ${ }^{1}$ Department of Mathematics, Faculty of Science, King Abdulaziz University, P.O. Box 80203, Jeddah 21589, Saudi Arabia \\ ${ }^{2}$ Laboratoire de Mathématiques, Images et Applications, Pôle Sciences et Technologies, Université de La Rochelle, Avenue M. Crepeau, \\ 17031 La Rochelle, France \\ ${ }^{3}$ Department of Mathematics, University College London, Gower Street, London WC1E 6BT, UK
}

Correspondence should be addressed to B. Ahmad; bashirahmad_qau@yahoo.com

Received 17 April 2014; Revised 9 June 2014; Accepted 9 June 2014; Published 23 June 2014

Academic Editor: Juan J. Nieto

Copyright (C) 2014 B. Ahmad et al. This is an open access article distributed under the Creative Commons Attribution License, which permits unrestricted use, distribution, and reproduction in any medium, provided the original work is properly cited.

The reaction diffusion system with anomalous diffusion and a balance law $u_{t}+(-\Delta)^{\alpha / 2} u=-f(u, v), v_{t}+(-\Delta)^{\beta / 2} v=f(u, v), 0<\alpha$, $\beta<2$, is con sidered. The existence of global solutions is proved in two situations: (i) a polynomial growth condition is imposed on the reaction term $f$ when $0<\alpha \leq \beta \leq 2$; (ii) no growth condition is imposed on the reaction term $f$ when $0<\beta \leq \alpha \leq 2$.

\section{Introduction}

In this paper, we consider the system of nonlinear and nonlocal in space reaction diffusion equations

$$
\begin{gathered}
u_{t}+(-\Delta)^{\alpha / 2} u=-f(u, v), \quad x \in \mathbb{R}^{N}, t>0, \\
v_{t}+(-\Delta)^{\beta / 2} v=f(u, v), \quad x \in \mathbb{R}^{N}, t>0,
\end{gathered}
$$

supplemented with the initial conditions

$$
u(x, 0)=u_{0}(x), \quad v(x, 0)=v_{0}(x), \quad x \in \mathbb{R}^{N},
$$

where the initial data $u_{0}(x), v_{0}(x)$ are given positive bounded functions.

Here the nonlocal operator $(-\Delta)^{\delta / 2}, \quad 0<\delta \leq 2(\delta=\alpha$ or $\beta$ ) accounts for anomalous diffusion (see, e.g., [1-3]) and can be defined via the Fourier transform pair $\mathscr{F}$ and $\mathscr{F}^{-1}$ as

$$
(-\Delta)^{\delta / 2} u(x)=\mathscr{F}^{-1}\left(|\xi|^{\delta} \mathscr{F}(u)(\xi)\right)(x), \quad u \in \mathcal{S}\left(\mathbb{R}^{N}\right)
$$

where $\mathcal{S}\left(\mathbb{R}^{N}\right)$ is the Schwartz class of smooth real rapidly decreasing functions, or equivalently (see [4]) by the formula

$$
(-\Delta)^{\delta / 2} u(x)=C_{N} P V \int_{\mathbb{R}^{N}} \frac{u(x)-u(y)}{|x-y|^{N+\delta}} d y
$$

with $C_{N}=\left(\delta 2^{\delta-1} / \pi^{N / 2}\right)(\Gamma((N+\delta) / 2) / \Gamma(1-(\delta / 2)))$ a normalizing constant, and $|\cdot|$ denotes the usual norm of $\mathbb{R}^{N}$.

A typical type of system under our consideration is given by the irreversible molecular combination

$$
m U+n V \longrightarrow(n+1) V,
$$

where $U$ and $V$ are two chemical species. If $u$ and $v$ represent the concentrations of the species $U$ and $V$, respectively, then according to the law of mass action due to Gulberg and Waage, the reaction diffusion system describing the chemical reaction can be written as

$$
\begin{aligned}
& u_{t}-\Delta u=-u^{m} v^{n}, \quad x \in \Omega, t>0, \\
& v_{t}-\Delta v=u^{m} v^{n}, \quad x \in \Omega, t>0,
\end{aligned}
$$

where $\Omega \subseteq \mathbb{R}^{N}$. This system has been studied by Masuda [5] via a judicious Lyapunov functional, Hollis et al. [6] by using the duality argument, Collet and Xin [7] in the case of the Euclidean space.

Let us now dwell for a while on the available literature concerning anomalous diffusion equations. Fractional differential equations have been used as effective mathematical tools for modeling diffusive processes associated with subdiffusion (fractional in time), superdiffusion (fractional in space), or both. Further examples can be found in physics, mathematical biology, or hydrology. These equations also 
appear in finance because of the relationship with certain option pricing mechanisms and heavy tailed stochastic processes [8]. In water resources, fractional models have been used to describe chemical and contaminant transport in heterogeneous aquifers [9]. In spatial complex environment, reaction diffusion equation may not obey Fick's Law [10]. One idea is to replace the flux, say $F$, by its fractional counterpart [11]:

$$
F=-\mathscr{K} \nabla^{\gamma} u, \quad 0<\gamma \leq 1,
$$

where $\mathscr{K}$ is the diffusion tensor and $\nabla^{\gamma}=$ $\left(\partial^{\gamma} / \partial x^{\gamma}, \partial^{\gamma} / \partial y^{\gamma}, \partial^{\gamma} / \partial z^{\gamma}\right)^{T}$ is the Riemann-Liouville fractional gradient, where

$$
\frac{\partial^{\gamma}}{\partial x^{\gamma}} u(x, y, z)=\frac{1}{\Gamma(1-\gamma)} \frac{\partial}{\partial x} \int_{0}^{x}(x-\tilde{x})^{-\gamma} u(\tilde{x}, y, z) d \tilde{x},
$$

with similar expressions for $\partial^{\gamma} / \partial y^{\gamma}$ and $\partial^{\gamma} / \partial z^{\gamma}$ [12]. The fractional Fick's Law for (8) implies nonlocality in space and in time. This modification, in the absence of external force, leads to the fractional diffusion equation

$$
\frac{\partial}{\partial t} u-\nabla \cdot\left(\mathscr{K} \nabla^{\gamma} u\right)=0
$$

Equivalently, in the isotropic setting [13], the space fractional reaction diffusion can be written as

$$
\frac{\partial}{\partial t} u+\mathscr{K}(-\Delta)^{\gamma / 2} u=f(u), \quad 0<\gamma \leq 1,
$$

where $(-\Delta)^{\gamma / 2}$ is the fractional Laplacian operator; see also the valuable contribution of Douglas [14] for the use of the fractional Laplacian in polymer sciences.

In our consideration, we take into account the diffusion of two interacting species, diffusing at different rates.

The reaction term $f:[0,+\infty)^{2} \rightarrow[0,+\infty)$ is locally Lipschitz continuous, namely,

$$
|f(u, v)-f(\widetilde{u}, \widetilde{v})| \leq C(R)(|u-\widetilde{u}|+|v-\widetilde{v}|),
$$

for all $0 \leq u, v, \widetilde{u}, \widetilde{v} \leq R$.

Further, it is assumed that there exist positive numbers $L(R), M(R)$, and $\kappa$ such that

$$
|f(u, v)| \leq L(R)|u|^{\kappa}+M(R),
$$

for all $u, v \geq 0$ with $|u| \leq R$, and

$$
\forall u, v \geq 0, \quad f(0, v)=0 \leq f(u, 0) .
$$

(Note that $f(u, v) \geq 0$ for all $u, v \geq 0$.)

We first prove that system (1)-(3) admits global solutions for reaction terms of polynomial growth relying on the duality argument that has been used by Hollis et al. [6] for the case when the space variable belongs to a bounded domain and $\alpha=\beta=2$. Notice that estimates obtained by this method have been recently improved by Cañizo et al. [15] in the same case $\alpha=\beta=2$. In case of $x \in \mathbb{R}^{N}$, the duality method has been used successfully by Fitzgibbon et al. [16] still in the case $\alpha=\beta=2$.

A central role in the proof is played by a recent $L^{p}$ regularity result due to Zhang [17] for the solution of the backward heat equation

$$
-\varphi_{t}+(-\Delta)^{\beta / 2} \varphi=\vartheta, \quad t \in(0, T), x \in \mathbb{R}^{N}, 0<\beta<2,
$$

supplemented with the condition

$$
\varphi(x, T)=0, \quad x \in \mathbb{R}^{N},
$$

which will be stated in Section 2 .

Next, we prove our second result; namely, global solutions of problem (1)-(3) exist for any growth of the reaction terms whenever $\beta \leq \alpha$.

Our second result has to be compared in some sense with that of Martin and Pierre [18]. It has been shown in [18] that the following problem admits global solutions for any nonlinearity $f$ under the condition $b \leq a$ :

$$
\begin{gathered}
u_{t}-a \Delta u=-f(u, v), \quad x \in \mathbb{R}^{N}, t>0, \\
v_{t}-b \Delta v=f(u, v), \quad x \in \mathbb{R}^{N}, t>0,
\end{gathered}
$$

supplemented with positive and bounded initial data.

The result of [18] is recalled in the appendix for the reader in order to compare our result with the result of Martin and Pierre.

The result of [18] has been extended by Kanel and Kirane [19] for the triangular system

$$
\begin{gathered}
u_{t}-a \Delta u=-f(u, v), \quad x \in \Omega, t>0, \\
v_{t}-c \Delta u-d \Delta v=f(u, v), \quad x \in \Omega, t>0, \\
\frac{\partial u}{\partial \nu}=\frac{\partial v}{\partial \nu}, \quad x \in \partial \Omega, t>0, \\
u(x, 0)=u_{0}(x), \quad u(x, 0)=u_{0}(x), \quad x \in \Omega,
\end{gathered}
$$

where $\Omega$ is a bounded regular domain with boundary $\partial \Omega, v$ is the outward normal derivative to $\partial \Omega$, and $a, c, d>0(a>d)$ are the positive diffusion constants.

\section{Preliminary Results}

Notation. Consider $Q_{T}=\mathbb{R}^{N} \times(0, T),\|u\|_{p}=$ $\left(\int_{\mathbb{R}^{N}}|u(x)|^{p} d x\right)^{1 / p}$, and $\|u\|_{p, T}=\left(\int_{Q_{T}}|u(x, t)|^{p} d x d t\right)^{1 / p}$.

The proof of our first result is based on a recent lemma of Zhang [17] (Lemma 2) and a known interpolation inequality (Lemma 3).

Lemma 1. Let $S_{\delta}(t):=e^{-t(-\Delta)^{\delta}}$ be the linear semigroup generated by the following linear anomalous diffusion problem:

$$
\begin{gathered}
\Psi_{t}+(-\Delta)^{\delta} \Psi=0, \quad(x, t) \in Q_{\infty}, \\
\Psi(x, 0)=\psi(x), \quad x \in \mathbb{R}^{N} .
\end{gathered}
$$


Let $1 \leq r \leq p \leq \infty$ and $\psi \in L^{r}\left(\mathbb{R}^{N}\right)$. Then the solution of (19) satisfies the estimate

$$
\left\|(-\Delta)^{\nu / 2} S_{\delta}(t) \psi(x)\right\|_{p} \leq C t^{-(\nu / 2 \delta)-(N / 2 \delta)((1 / r)-(1 / p))}\|\psi\|_{r}
$$

for $\delta>0$ and $v \geq 0$.

The proof of this lemma follows from the Young inequality combined with scaling properties of the kernel

$$
\mathscr{K}(x, t)=(2 \pi)^{N / 2} \int_{\mathbb{R}^{N}} e^{i\langle x, \xi\rangle-t|\xi|^{2 \delta}} d \xi=t^{-(N / 2 \delta)} \mathscr{K}\left(\frac{x}{t^{1 / 2 \delta}}\right)
$$

with

$$
\mathscr{K}=(2 \pi)^{N / 2} \int_{\mathbb{R}^{N}} e^{i\langle x, \xi\rangle-|\xi|^{2 \delta}} d \xi,
$$

where $\langle x, \xi\rangle$ is the ordinary inner product at the points $x, \xi \in$ $\mathbb{R}^{N}$ and $|\xi|^{2}=\langle\xi, \xi\rangle$.

The lemma is used for the local existence $(\nu=0)$, as well as for the global existence $(\nu \geq 0)$.

Lemma 2. Let $1<q<\infty$ and suppose that $\vartheta \in L^{q}\left(Q_{T}\right)$. Then (15)-(16) has a unique positive solution $\varphi$ such that $\varphi_{t} \in$ $L^{q}\left(Q_{T}\right),(-\Delta)^{\beta / 2} \varphi \in L^{q}\left(Q_{T}\right)$. Moreover, there exists a constant $C(p, T)$, independent of $\varphi$ such that

$$
\|\varphi\|_{q, T}+\|\varphi\|_{q}+\left\|(-\Delta)^{\beta / 2} \varphi\right\|_{q, T}+\left\|\varphi_{0}\right\|_{q} \leq C\|\vartheta\|_{q, T} .
$$

Lemma 3. Let $(\mathscr{X},\|\cdot\|)$ be a Banach space and A a positive operator on $\mathscr{X}$. Then, for $0 \leq \varrho<\sigma<\gamma$, there exists a constant $C_{\varrho, \sigma, \gamma}$ such that for $x \in D\left(A^{\gamma}\right)$ (the domain of $A^{\gamma}$ )

$$
\left\|A^{\sigma} x\right\| \leq C_{\varrho, \sigma, \gamma}\left\|A^{\gamma} x\right\|^{(\sigma-\varrho) /(\gamma-\varrho)}\left\|A^{\varrho} x\right\|^{(\gamma-\sigma) /(\gamma-\varrho)} .
$$

The proof of our second result is based on the following interesting lemma of Lopez-Mimbela and Morales [20].

Let $f_{\delta}(x, t)$ be the continuous transition density of the symmetric stable process $\{X(t) ; t \geq 0\}$ in $\mathbb{R}^{N}$ of index $\delta$, $0<\delta \leq 2$, which is uniquely determined by

$$
e^{-t|\xi|^{\delta}}=\int_{\mathbb{R}^{N}} e^{i\langle x, \xi\rangle} f_{\delta}(x, t) d x .
$$

Lemma 4. Let $f_{\alpha_{i}}(x, t), t>0$ be the transition density of the symmetric $\alpha_{i}$-stable process in $\mathbb{R}^{N}, i=1,2$. If $\alpha_{1} \leq \alpha_{2}$, then there exists a constant $K \geq 1$ such that, for every $t>0$ and $x \in \mathbb{R}^{N}$,

$$
f_{\alpha_{i}}(x, t) \leq K f_{\alpha_{1}}\left(x, t^{\alpha_{1} / \alpha_{i}}\right), \quad i=1,2 .
$$

If in addition $t \geq 1$, then

$$
\begin{aligned}
f_{\alpha_{i}}(x, t) & =K t^{N\left(\left(1 / \alpha_{1}\right)-\left(1 / \alpha_{2}\right)\right)} f_{\alpha_{1}}(x, t) \\
& \leq K t^{N / \alpha_{1}} f_{\alpha_{1}}(x, t), \quad x \in \mathbb{R}^{N} .
\end{aligned}
$$

As the proof is nice and instructive, we present it for the convenience of the reader.

Proof. By Theorem 2.1 [21], we have

$$
\begin{aligned}
& \lim _{|x| \rightarrow \infty}|x|^{N+\alpha_{i}} f_{\alpha_{i}}(x, 1) \\
& =\alpha_{i} 2^{\alpha-1}\left(\frac{1}{\pi}\right)^{(N / 2)+1} \sin \left(\frac{\alpha_{i} \pi}{2}\right) \Gamma\left(\frac{\alpha_{i}}{2}\right) \Gamma\left(\frac{N+\alpha_{i}}{2}\right) \\
& =C_{\alpha_{i}}>0, \quad\left(\alpha_{i}<2\right) .
\end{aligned}
$$

If $\alpha_{1}<\alpha_{2}$, then $f_{\alpha_{2}}(x, 1) / f_{\alpha_{1}}(x, 1) \sim\left(C_{\alpha_{2}} / C_{\alpha_{1}}\right)|x|^{\alpha_{1}-\alpha_{2}} \rightarrow 0$ as $|x| \rightarrow \infty$. Hence, there exists a constant $C>0$ such that $f_{\alpha_{2}}(x, 1) / f_{\alpha_{1}}(x, 1) \leq 1$ for all $|x|>C$. Since $f_{\alpha_{2}}(x, 1) / f_{\alpha_{1}}(x, 1)$ is continuous and $\left\{y \in \mathbb{R}^{N} ;|y| \leq C\right\}$ is compact, there exists $M>0$ such that $f_{\alpha_{2}}(x, 1) / f_{\alpha_{1}}(x, 1) \leq M$ for all $|x| \leq C$. Thus $f_{\alpha_{2}}(x, 1) \leq K f_{\alpha_{1}}(x, 1)$ for all $x \in \mathbb{R}^{N}$, where $K=\max \{1, M\}$. From scaling properties of stable densities, we get

$$
\begin{aligned}
f_{\alpha_{2}}(x, t) & =t^{-N / \alpha_{2}} f_{\alpha_{2}}\left(x t^{-1 / \alpha_{2}}, 1\right) \\
& \leq t^{-N / \alpha_{2}} K f_{\alpha_{1}}\left(x t^{-1 / \alpha_{2}}, 1\right) \\
& =t^{-N / \alpha_{2}} K\left(t^{\alpha_{1} / \alpha_{2}}\right)^{N / \alpha_{1}} f_{\alpha_{1}}\left(x, t^{\alpha_{1} / \alpha_{2}}\right) \\
& =K f_{\alpha_{1}}\left(x, t^{\alpha_{1} / \alpha_{2}}\right),
\end{aligned}
$$

which is (26).

Now assume that $t \geq 1$. Using (29) and the fact that $f_{\alpha_{1}}(x, 1)$ is radially decreasing, we may write

$$
\begin{aligned}
f_{\alpha_{2}}(x, t) & \leq K t^{-N / \alpha_{2}} f_{\alpha_{1}}\left(x t^{-1 / \alpha_{2}}, 1\right) \\
& =K t^{N\left(\left(1 / \alpha_{1}\right)-\left(1 / \alpha_{2}\right)\right)}\left(t^{-\left(N / \alpha_{1}\right)} f_{\alpha_{1}}\left(x t^{-\left(1 / \alpha_{2}\right)}, 1\right)\right) \\
& \leq K t^{N\left(\left(1 / \alpha_{1}\right)-\left(1 / \alpha_{2}\right)\right)} f_{\alpha_{1}}(x, t) .
\end{aligned}
$$

\section{Main Results}

Now, we are ready to announce and prove our main results.

Local existence of a classical nonnegative solution $(u, v)$ of (1)-(3) on a maximal interval of existence $\left[0, T_{\max }\right)$ is obtained as usual (see, e.g., [22]).

Theorem 5. Assume $u_{0}, v_{0} \in L^{\infty}\left(\mathbb{R}^{N}\right), u_{0}, v_{0} \geq 0$ a.e. on $\mathbb{R}^{N}$. Let the nonlinearity $f$ satisfy (12), (14), and the polynomial growth condition (13). Then problem (1)-(3) admits a nonnegative classical solution on $\mathbb{R}^{N} \times(0,+\infty)$.

Proof. First, as $u_{0} \geq 0, v_{0} \geq 0$ and $f$ satisfies condition (14), we have $u \geq 0$ and $v \geq 0$.

In view of the maximum principle, we have the estimate

$$
\|u\|_{p, T} \leq\left\|u_{0}\right\|_{p, T} .
$$

Case $1(0<\alpha \leq \beta \leq 2)$. From (1) and (2), we have

$$
u_{t}+v_{t}+(-\Delta)^{\alpha / 2}(u)+(-\Delta)^{\beta / 2}(v)=0,
$$


which can alternatively be rewritten as

$$
(u+v)_{t}+(-\Delta)^{\beta / 2}(u+v)+(-\Delta)^{\alpha / 2}(u)-(-\Delta)^{\beta / 2}(u)=0 .
$$

Now, we use the duality argument. By multiplying (33) throughout by $\varphi$, the solution of (15)-(16), and integrating by parts over $Q_{T}$, we obtain

$$
\begin{aligned}
\int_{Q_{T}}(u+v)\left(-\varphi_{t}+(-\Delta)^{\beta / 2}(\varphi)\right) \\
=\int_{\mathbb{R}^{N}}\left(u_{0}+v_{0}\right) \varphi(x, 0) \\
\quad+\int_{Q_{T}} u\left((-\Delta)^{\beta / 2}(\varphi)-(-\Delta)^{\alpha / 2}(\varphi)\right)
\end{aligned}
$$

or

$$
\begin{aligned}
\int_{\mathrm{Q}_{T}}(u+v) \vartheta= & \int_{\mathbb{R}^{N}}\left(u_{0}+v_{0}\right) \varphi(x, 0) \\
& +\int_{\mathrm{Q}_{T}} u\left((-\Delta)^{\beta / 2}(\varphi)-(-\Delta)^{\alpha / 2}(\varphi)\right) .
\end{aligned}
$$

Using Lemma 2, we have

$$
\begin{gathered}
\|\varphi\|_{q}+\|\varphi\|_{q, T} \leq C\|\vartheta\|_{q, T}, \\
\left\|(-\Delta)^{\beta / 2} \varphi\right\|_{q, T} \leq C\|\vartheta\|_{q, T} .
\end{gathered}
$$

Making use of inequality (24) together with $\varrho=0, \sigma=\alpha / 2$, and $\gamma=\beta / 2$, we obtain

$$
\left\|(-\Delta)^{\alpha / 2} \varphi\right\|_{q} \leq C\left\|(-\Delta)^{\beta / 2} \varphi\right\|_{q}^{\alpha / \beta}\|\varphi\|_{q}^{1-(\alpha / \beta)} .
$$

Using estimates (36) and (37), we have

$$
\begin{aligned}
\left\|(-\Delta)^{\alpha / 2} \varphi\right\|_{q, T} & \leq C\left(\|\vartheta\|_{q, T}\right)^{\alpha / \beta}\left(\|\vartheta\|_{q, T}\right)^{1-(\alpha / \beta)} \\
& \leq C\|\vartheta\|_{q, T} .
\end{aligned}
$$

Now, we have the estimates

$$
\begin{aligned}
\int_{Q_{T}} u\left|(-\Delta)^{\beta / 2}(\varphi)\right| & \leq\|u\|_{p, T}+\left\|(-\Delta)^{\beta / 2}(\varphi)\right\|_{q, T}, \\
p+q=p q, & \\
\int_{Q_{T}} u\left|(-\Delta)^{\alpha / 2}(\varphi)\right| & \leq\left(\|u\|_{p, T}+\left\|(-\Delta)^{\alpha / 2}(\varphi)\right\|_{q, T}\right) \\
& \leq C\left(\|u\|_{p, T}+\|\vartheta\|_{q, T}\right), \quad p+q=p q .
\end{aligned}
$$

Finally, we have

$$
\int_{Q_{T}}(u+v) \vartheta \leq C\left(\left\|u_{0}+v_{0}\right\|_{p}+\|u\|_{p, T}+\|\vartheta\|_{q, T}+\left\|\varphi_{0}\right\|_{q}\right)
$$

thanks to the above inequalities.
Since $\vartheta$ is arbitrarily nonnegative in $L^{q}\left(Q_{T}\right)$ and $u, v \geq 0$, therefore it follows by duality that

$$
\|u+v\|_{p, T} \leq C\left(\left\|u_{0}+v_{0}\right\|_{p}+\|u\|_{p, T}+\|\vartheta\|_{q, T}+\left\|\varphi_{0}\right\|_{q}\right) .
$$

Therefore, for all $p<\infty$, the $L^{p}$-norm of $u$ and $v$ remains finite on $Q_{T_{\max }}$. From the polynomial growth assumption on the nonlinearity, it follows that $f(u, v)$ is also in $L^{p}\left(Q_{T_{\max }}\right)$ for all $p \in(1, \infty)$. If we take $p>(N+1) / 2$, we deduce that $v \in L^{p}\left(Q_{T_{\max }}\right)$ :

$$
\|v\|_{\infty, T_{\max }} \leq C\|f(u, v)\|_{p, T_{\max }}<+\infty .
$$

This implies that $T_{\max }=\infty$.

Case $2(0<\beta \leq \alpha \leq 2)$. This case can be treated in the same way by making use of inequality (20) with $\nu=1$.

The next theorem deals with the "no growth" restriction on $f$.

Theorem 6. Assume $0<\beta \leq \alpha \leq 2$ and $u_{0}, v_{0} \in L^{\infty}\left(\mathbb{R}^{N}\right)$, $u_{0}, v_{0} \geq 0$ a.e. on $\mathbb{R}^{N}$. Let the nonlinearity $f$ satisfy (12) and (14). Then problem (1)-(3) has a classical solution on $\mathbb{R}^{N} \times$ $(0,+\infty)$.

Proof. Let $S_{\delta}(t)$ be the semigroup generated by $(-\Delta)^{\delta / 2}$ on $\mathbb{R}^{N}$. Then we have

$$
\begin{gathered}
u(x, t)=S_{\alpha}(t) u_{0}(x)-\mathfrak{F}_{\alpha}(x, t), \\
v(x, t)=S_{\beta}(t) v_{0}(x)+\mathfrak{F}_{\beta}(x, t),
\end{gathered}
$$

where $\mathfrak{F}_{\delta}(x, t)=\int_{0}^{t} \int_{\mathbb{R}^{N}} f_{\delta}(y, s) u(x-y, t-s) f(v(x-y, t-$ s)) $d y d s, \delta=\alpha$, or $\delta=\beta$.

From (26), we have

$$
0 \leq \mathfrak{F}_{\alpha}(x, t) \leq S_{\alpha}(t) u_{0}(x) \leq C\left\|u_{0}\right\|_{\infty} .
$$

Using Lemma 1 for $t \geq 1$, we obtain

$$
\begin{aligned}
& \mathfrak{F}_{\beta}(x, t)= \int_{0}^{t} \int_{\mathbb{R}^{N}} f_{\beta}(y, s) u(x-y, t-s) \\
& \times f(v(x-y, t-s)) d y d s \\
& \leq K t^{N / \alpha} \int_{0}^{t} \int_{\mathbb{R}^{N}} f_{\beta}(y, s) u(x-y, t-s) \times f(v(x-y, t-s)) d y d s \\
& \leq K t^{N / \alpha} \mathfrak{F}_{\alpha}(t, x) \leq C K t^{N / \alpha}\left\|u_{0}\right\|_{\infty} .
\end{aligned}
$$

So $\mathfrak{F}_{\beta}(t, x)$ is bounded for any finite $t$, whereupon the solution is global.

Remark 7. Our results remain valid when the reaction terms $f(u, v)$ in the first equation and $g(u, v)$ in the second equation satisfy

$$
f(u, v)+g(u, v) \leq L(u+v)+M, \quad \forall u, v \geq 0,
$$

where $L$ and $M$ are nonnegative constants. 


\section{Appendix}

Here we present the result of Martin and Pierre [18] concerning the determination of the bound on the component $v$ of the system

$$
\begin{gathered}
\frac{\partial}{\partial t} u-a \Delta u=-f(u, v), \quad(x, t) \in \mathbb{R}^{N} \times(0, \infty), \\
\frac{\partial}{\partial t} v-b \Delta v=+f(u, v), \quad(x, t) \in \mathbb{R}^{N} \times(0, \infty), \\
u(\cdot, 0)=u_{0}(\cdot), \quad v(\cdot, 0)=v_{0}(\cdot), \quad x \in \mathbb{R}^{N},
\end{gathered}
$$

where $f$ satisfies hypotheses (12) and (14).

Theorem 8. Assume

$$
\begin{gathered}
b \leq a, \\
u_{0}, v_{0} \in L^{\infty}\left(\mathbb{R}^{N}\right), \quad u_{0}, v_{0} \geq 0 \text { a.e } x \in \mathbb{R}^{N} .
\end{gathered}
$$

Then (A.1) has a classical solution on $\mathbb{R}^{N} \times(0, \infty)$.

Proof. For $t \in\left(0, T_{\max }\right)$, we can write

$$
\begin{aligned}
& u(t)=S_{a}(t) u_{0}-\int_{0}^{t} S_{a}(t-s) f(u, v)(s) d s \\
& v(t)=S_{b}(t) u_{0}+\int_{0}^{t} S_{b}(t-s) f(u, v)(s) d s
\end{aligned}
$$

via the semigroups $S_{a}(t)$ and $S_{b}(t)$, where

$$
S_{\lambda}(t) u_{0}(x)=(4 \pi \lambda t)^{-N / 2} \int_{\mathbb{R}^{N}} \exp \left(-\frac{|x-y|^{2}}{4 \lambda t}\right) u_{0}(y) d y .
$$

It is not difficult to see that

$$
\begin{aligned}
& \int_{0}^{t} S_{b}(t-s) f(u, v)(s) d s \\
& \quad \leq\left(\frac{a}{b}\right)^{N / 2} \int_{0}^{t} S_{a}(t-s) f(u, v)(s) d s .
\end{aligned}
$$

From (A.3)-(A.6) and $u \geq 0$, we deduce

$$
v(t) \leq S_{b}(t) v_{0}+\left(\frac{a}{b}\right)^{N / 2} S_{a}(t) u_{0},
$$

which provides a uniform $L^{\infty}$-bound for $v$.

\section{Conflict of Interests}

The authors declare that there is no conflict of interests regarding the publication of this paper.

\section{Acknowledgment}

This project was funded by the Deanship of Scientific Research (DSR), King Abdulaziz University, Jeddah, under Grant no. 21/34/GR. The authors, therefore, acknowledge with thanks the DSR technical and financial support.

\section{References}

[1] M. F. Schlesinger, G. M. Zaslavsky, and U. Frisch, Lévy Flights and Related Topics in Physics, Springer, Berlin, Germany, 1994.

[2] J. Klafter, M. F. Shlesinger, and G. Zumofen, "Beyond Brownian motion," Physics Today, vol. 49, no. 2, pp. 33-39, 1996.

[3] T. H. Solomon, E. R. Weeks, and H. L. Swinney, "Observation of anomalous diffusion and Lévy flights in a two-dimensional rotating flow," Physics Reports, vol. 339, pp. 1-77, 1993.

[4] N. S. Landkof, Foundations of Modern Potential Theory, Springer, New York, NY, USA, 1972.

[5] K. Masuda, "On the global existence and asymptotic behavior of solutions of reaction-diffusion equations," Hokkaido Mathematical Journal, vol. 12, no. 3, pp. 360-370, 1983.

[6] S. L. Hollis, R. H. Martin, Jr., and M. Pierre, "Global existence and boundedness in reaction-diffusion systems," SIAM Journal on Mathematical Analysis, vol. 18, no. 3, pp. 744-761, 1987.

[7] P. Collet and J. Xin, "Global existence and large time asymptotic bounds of $L^{\infty}$ solutions of thermal diffusive combustion systems on $R^{n}$," Annali della Scuola Normale Superiore di Pisa: Classe di Scienze Serie IV, vol. 23, no. 4, pp. 625-642, 1996.

[8] E. Scalas, R. Gorenflo, and F. Mainardi, "Fractional calculus and continuous-time finance," Physica A: Statistical Mechanics and Its Applications, vol. 284, no. 1, pp. 376-384, 2000.

[9] D. A. Benson, S. W. Wheatcraft, and M. M. Meerschaert, "Application of a fractional advection-dispersion equation," Water Resources Research, vol. 36, no. 6, pp. 1403-1412, 2000.

[10] Y. Zhang, D. A. Benson, and D. M. Reeves, "Time and space nonlocalities underlying fractional-derivative models: distinction and literature review of field applications," Advances in Water Resources, vol. 32, no. 4, pp. 561-581, 2009.

[11] M. M. Meerschaert, "Fractional calculus modelsfor anomalous diffusion," SAMSI Summer Program on Nonlocal Continuum Models for Iffusion, Mechanics, and Other Applications, 2012, http://www.samsi.info/sites/default/files/Meerschaert.

[12] S. G. Samko, A. A. Kilbas, and O. I. Marichev, Integrals and Derivatives: Theory and Applications, Gordon and Breach Science, Yverdon, Switzerland, 1993.

[13] I. Turner, M. Ilic, and P. Perre, "The use of fractiona-inspace diffusion equations for describing miscoscale diffusion in porous media," in Proceedings of the International Conference, Magdebourg, Germany, 2010.

[14] J. F. Douglas, "Polymer science applications of path-integration, integral equations, and fractional calculus," in Applications of Fractional Calculus in Physics, R. Hilfer, Ed., pp. 241-330, World Scientifiv, River Edge, NJ, USA, 2000.

[15] J. A. Cañizo, L. Desvillettes, and K. Fellner, "Improved duality estimates and applications to reaction-diffusion equations," Communications in Partial Differential Equations, vol. 39, no. 6, pp. 1185-1204, 2014.

[16] W. E. Fitzgibbon, J. Morgan, and R. Sanders, "Global existence and boundedness for a class of inhomogeneous semilinear parabolic systems," Nonlinear Analysis: Theory, Methods \& Applications, vol. 19, no. 9, pp. 885-899, 1992.

[17] X. Zhang, " $L^{p}$-maximal regularity of nonlocal parabolic equations and applications," Annales de l'Institut Henri Poincaré: Analyse Non Linéaire, vol. 30, no. 4, pp. 573-614, 2013.

[18] R. H. Martin and M. Pierre, "Nonlinear reaction-diffusion systems," in Nonlinear Equations in the Applied Sciences, W. F. Ames and C. Rogers, Eds., Academic Press, 1990. 
[19] J. I. Kanel and M. Kirane, "Global existence and large time behavior of positive solutions to a reaction diffusion system," Differential and Integral Equations, vol. 13, no. 1-3, pp. 255-264, 2000.

[20] J. A. Lopez-Mimbela and J. V. Morales, "Local time and Tanaka formula for a multitype Dawson-Watanabe super-process," Mathematische Nachrichten, vol. 279, no. 15, pp. 1695-1708, 2006.

[21] R. M. Blumenthal and R. K. Getoor, "Some theorems on stable processes," Transactions of the American Mathematical Society, vol. 95, pp. 263-273, 1960.

[22] A. Fino and M. Kirane, "Qualitative properties of solutions to a nonlocal evolution system," Mathematical Methods in the Applied Sciences, vol. 34, no. 9, pp. 1125-1143, 2011. 


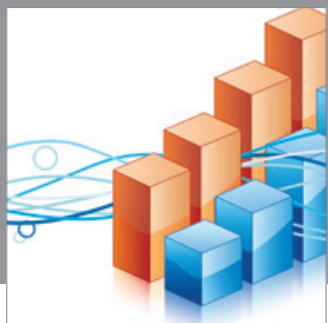

Advances in

Operations Research

mansans

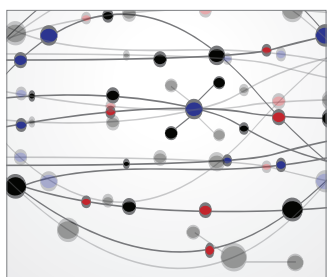

The Scientific World Journal
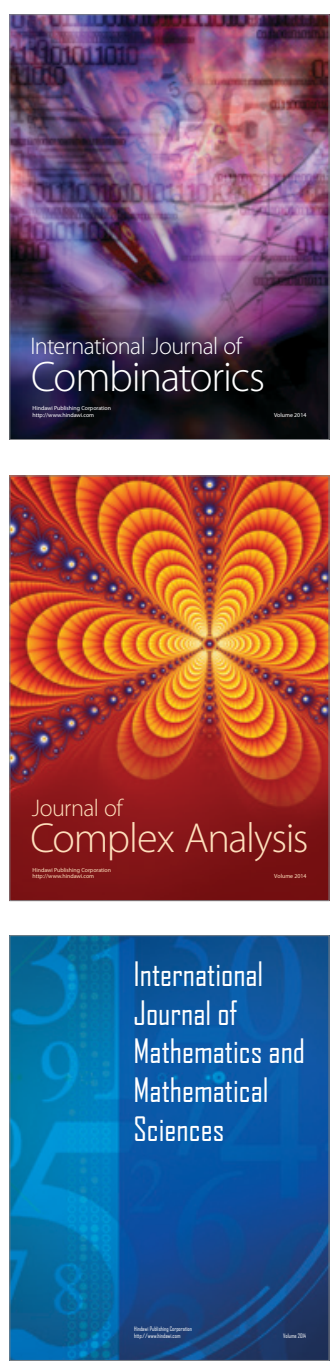
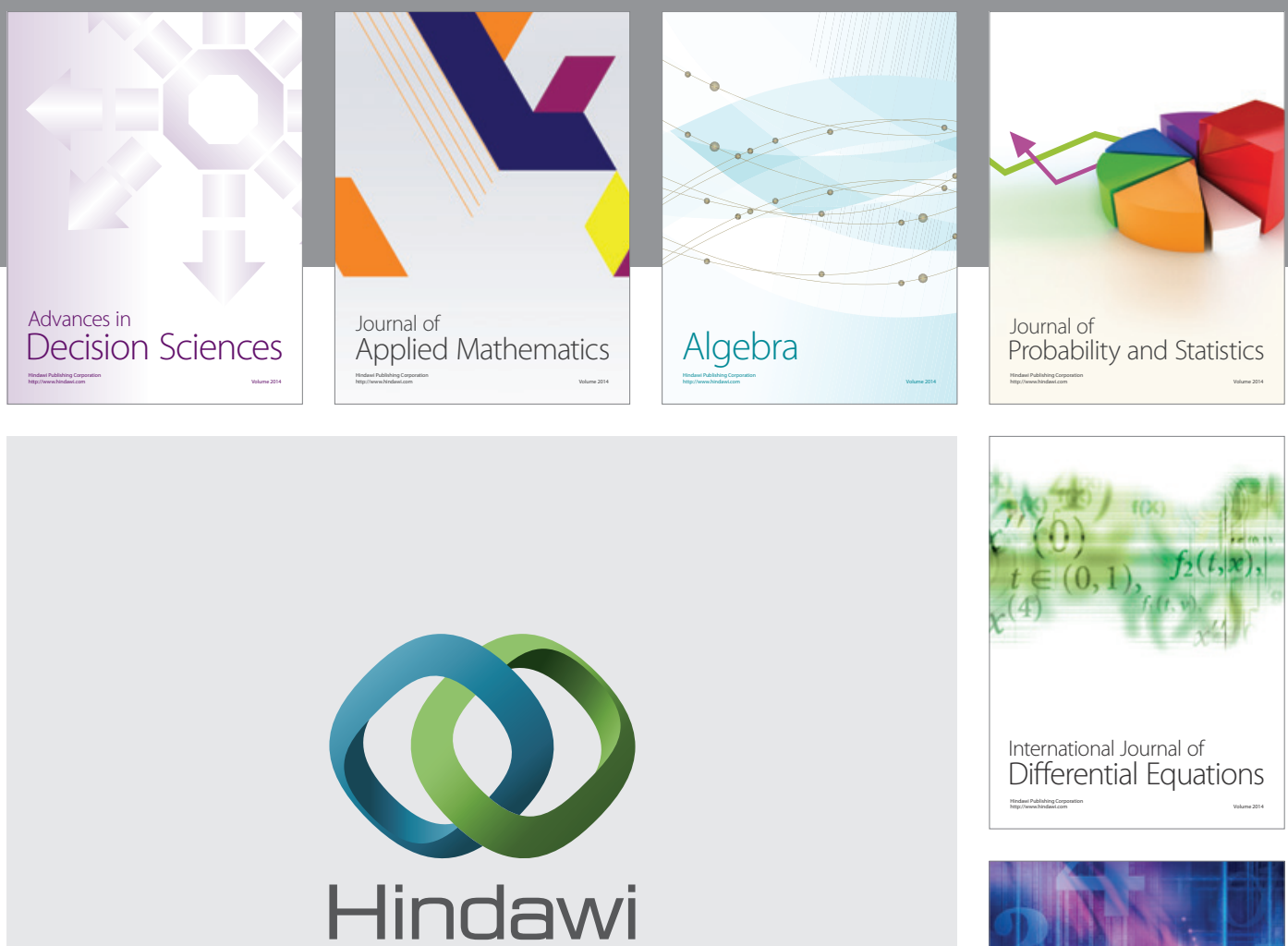

Submit your manuscripts at http://www.hindawi.com
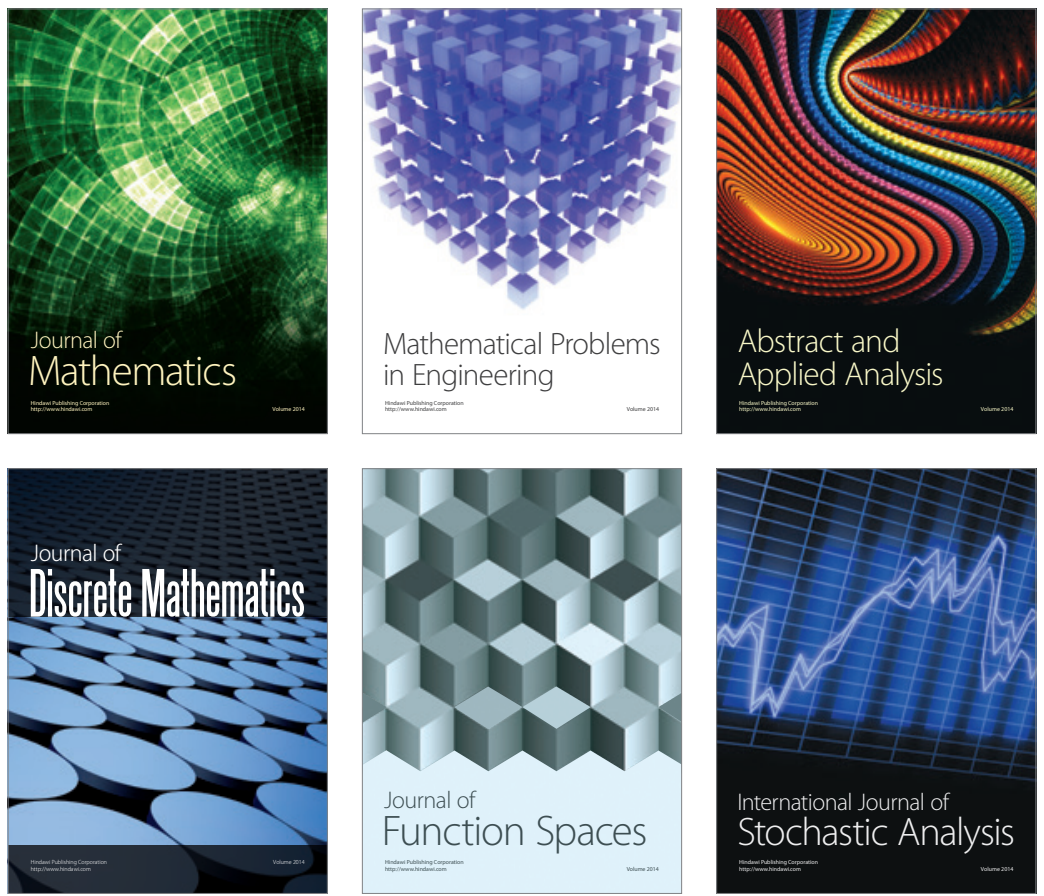

Journal of

Function Spaces

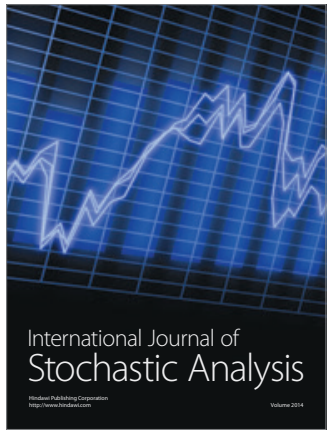

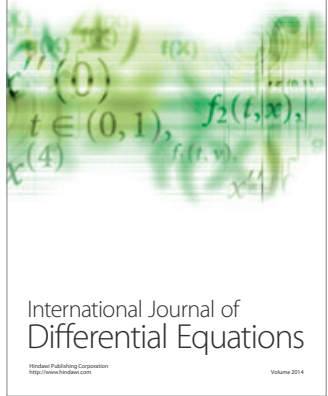
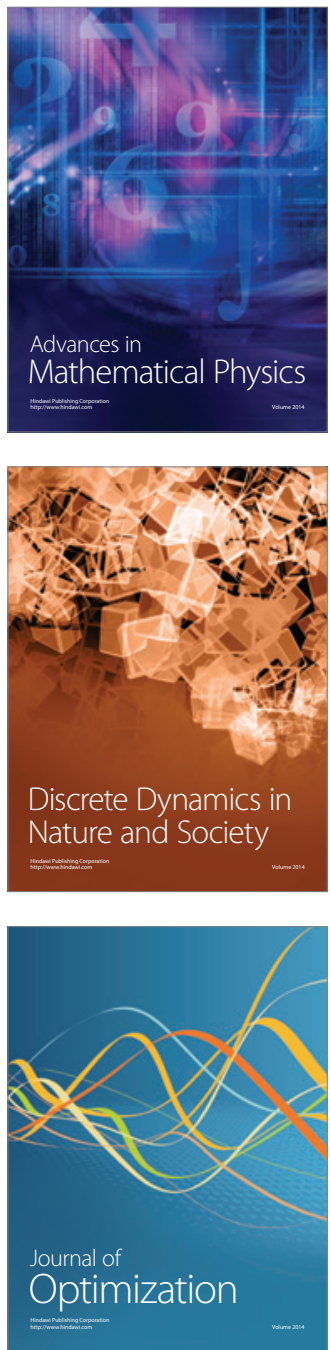